\title{
Linear stability analysis and validation of a unified solution method for fluid-structure-interaction on structural dynamic problems
}

\section{Citation for published version (APA):}

Giannopapa, C. G., \& Papadakis, G. (2005). Linear stability analysis and validation of a unified solution method for fluid-structure-interaction on structural dynamic problems. (CASA-report; Vol. 0510). Technische Universiteit Eindhoven.

\section{Document status and date:}

Published: 01/01/2005

\section{Document Version:}

Publisher's PDF, also known as Version of Record (includes final page, issue and volume numbers)

\section{Please check the document version of this publication:}

- A submitted manuscript is the version of the article upon submission and before peer-review. There can be important differences between the submitted version and the official published version of record. People interested in the research are advised to contact the author for the final version of the publication, or visit the DOI to the publisher's website.

- The final author version and the galley proof are versions of the publication after peer review.

- The final published version features the final layout of the paper including the volume, issue and page numbers.

Link to publication

\footnotetext{
General rights

- You may freely distribute the URL identifying the publication in the public portal. follow below link for the End User Agreement:

www.tue.nl/taverne

Take down policy

If you believe that this document breaches copyright please contact us at:

openaccess@tue.nl

providing details and we will investigate your claim.
}

Copyright and moral rights for the publications made accessible in the public portal are retained by the authors and/or other copyright owners and it is a condition of accessing publications that users recognise and abide by the legal requirements associated with these rights.

- Users may download and print one copy of any publication from the public portal for the purpose of private study or research.

- You may not further distribute the material or use it for any profit-making activity or commercial gain

If the publication is distributed under the terms of Article 25fa of the Dutch Copyright Act, indicated by the "Taverne" license above, please 


\title{
LINEAR STABILITY ANALYSIS AND VALIDATION OF A UNIFIED SOLUTION METHOD FOR FLUID-STRUCTURE-INTERACTION ON STRUCTURAL DYNAMIC PROBLEMS
}

\author{
C. G. Giannopapa \\ Dept. of Mathematics and Computer Science \\ Technische Universiteit Eindhoven \\ PO Box 513, 5600 MB Eindhoven \\ The Netherlands \\ Email: c.g.giannopapa@tue.nl
}

\author{
G. Papadakis \\ Experimental and computational laboratory for \\ the analysis of turbulence \\ Department of Mechanical Engineering \\ King's College London, Strand WC2R 2LS, UK \\ Email: george.papadakis@kcl.ac.uk
}

\begin{abstract}
In the conventional approach for fluid-structure interaction problems, the fluid and solid components are treated separately and information is exchanged across their interface. According to the conventional terminology, the current numerical methods can be grouped in two major categories: Partitioned methods and monolithic methods. Both methods use two separate sets of equations for fluid and solid. A unified solution method has been presented [1], which is different from these methods. The new method treats both fluid and solid as a single continuum, thus the whole computational domain is treated as one entity discretised on a single grid. Its behavior is described by a single set of equations, which are solved fully implicitly. In this paper, 2 time marching and one spatial discretisation scheme, widely used for fluids' equations, are applied for the solution of the equations for solids. Using linear stability analysis, the accuracy and dissipation characteristics of the resulting difference equations are examined. The aforementioned schemes are applied to a transient structural problem (beam bending) and the results compare favorably with available analytic solutions and are consistent with the conclusions of the stability analysis. A parametric investigation using different meshes, time steps and beam sizes is also presented. For all cases examined the numerical solution was stable and robust and proved to be suitable for the next stage of application to full fluid-structure interaction problems.
\end{abstract}

\section{NOMENCLATURE}

\begin{tabular}{lll}
\multicolumn{3}{l}{ Roman symbols } \\
Co & - & Courant number \\
$D$ & $\mathrm{~m}$ & displacement \\
$f$ & $\mathrm{~Hz}$ & frequency \\
$h$ & $\mathrm{~m}$ & height \\
$I$ & - & unit tensor \\
$K$ & $\mathrm{~Pa}$ & bulk modulus \\
$L$ & $\mathrm{~m}$ & length \\
$\mathrm{F}$ & - & fluid \\
$\mathrm{p}$ & $\mathrm{Pa}$ & pressure \\
$\mathrm{S}$ & - & solid \\
$t$ & $\mathrm{~s}$ & time \\
$U$ & $\mathrm{~m} / \mathrm{s}$ & velocity
\end{tabular}




\begin{tabular}{lll}
\multicolumn{3}{l}{ Greek symbols } \\
$\Delta t$ & $\mathrm{~s}$ & time step \\
$\Delta x$ & $\mathrm{~m}$ & grid spacing \\
$\eta$ & $\mathrm{m}^{2} / \mathrm{s}$ & dynamic viscosity \\
$\lambda$ & $\mathrm{Pa}$ & Lame's coefficient \\
$\mu$ & $\mathrm{Pa}$ & Lame's coefficient \\
$\varepsilon$ & - & strain tensor \\
$E$ & $\mathrm{~Pa}$ & Young's modulus \\
$\nu$ & - & Poison ratio \\
$\rho$ & $\mathrm{kg} / \mathrm{m}^{3}$ & density \\
$\sigma$ & $\mathrm{Pa}$ & Cauchy stress tensor \\
$\tau$ & $\mathrm{Pa}$ & applied end shear \\
$\omega$ & $\mathrm{Hz}$ & frequency of undamped oscillations
\end{tabular}

\section{INTRODUCTION}

Fluid structure interaction (FSI) occurs in many areas of engineering (aerospace, civil or mechanical) as well as other scientific disciplines including medicine, biomechanics etc. The term FSI describes the influence of a fluid variable (for example pressure or temperature) on a solid and crucially vice versa. This interaction between the two components may be due to thermal transfer or momentum transfer or often a combination of the two. This paper is concerned with the interaction between a solid body and a fluid due to momentum transfer only. More specifically, is concerned with the local deformations of the solid rather than the bulk deformation and rigid body motion [2].

During this interaction, the fluid normal and shear stress act on the structure and cause it to deform, which in turn affects the fluid flow and consequently the stress field of the fluid. Thus, the response of the system can be determined only if the fully coupled problem is solved. This interaction can have an enormous effect on the speed of pressure wave propagation and becomes particularly important when the liquid is almost incompressible, in which case the deformation on the solid cannot be neglected [3]. The reliable prediction of pressure waves is important in liquid filled vessels such as arterial flow, impact on liquid filled containers, pipelines etc.

The different numerical techniques for the solution of the equations describing an FSI problem are classified according to the way time is treated. Numerical methods can be grouped in three major categories: partitioned methods, monolithic methods and single solution methods. Figure 1 below assists the reader with the conceptual understanding of the single solution method and its differences from the conventional methods.

\section{Partitioned methods}

In the partitioned methods, shown schematically in Figure 1a, the fluid and solid solution domains are partitioned, the equations for both components are solved alternatively and the enforcement of kinematic and dynamic conditions at the interface is asynchronous. Typically two separate software packages are used for modeling the solid and fluid components. Partitioning leads inherently to loss of conservation of properties of the continua (fluid and structure). The energy increase in the system leads to instability, which is the major drawback of the method. An overview of the benefits and disadvantages of these methods can be found in [4]. 
(a)

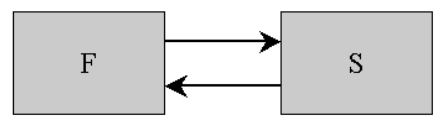

(b)

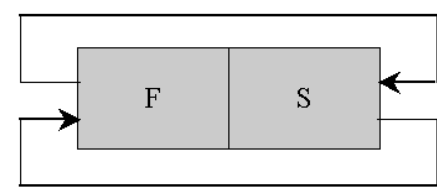

(c)

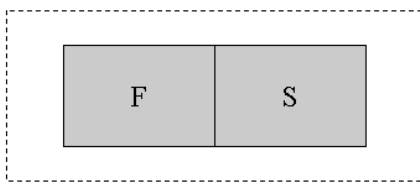

Figure 1: Classification of FSI methods
Partitioned

method

Monolithic

method

Single solution

method

\section{Monolithic methods}

The monolithic methods, shown in Figure 1b, use two separate sets of equations for fluid and solid, couple the fluid dynamics and structural dynamics implicitly and solve them synchronously at their common interface [5]. These methods can be unconditionally stable and energy conserving when the modified Osher scheme is used for the fluid elements [6].

\section{Single solution method}

The single solution method [1,7] used in this paper is a fully coupled method in which the fluid and solid are solved simultaneously for each time step in a single solution domain (Figure 1c). A single set of equations is used to describe the fluid and solid and their interface is contained within the solution domain itself making the coupling inherently implicit. The objective is to model fluid and solid simultaneously in a single solution environment and thus to eliminate stability problems associated with the segregated approach.

\section{MATHEMATICAL FORMULATION}

This paragraph is concerned with the presentation of the mathematical formulation of the unified method for the solution of the equations for solids and liquids as described in [1,7]. The resulting set of equations contains the three velocity components and pressure as unknown variables. The deformations are assumed to be small.

The following equations describe continuum media and are thus valid for both solids and fluids.

Momentum equation (neglecting body forces):

$\frac{\partial \rho U}{\partial t}+\nabla \cdot(\rho U U)=\nabla \cdot \sigma$

Continuity equation (or mass conservation):

$\frac{\partial \rho}{\partial t}+\nabla \cdot(\rho U)=0$

The difference between solid and fluid lies in the constitutive equation for the stress tensor, $\sigma$. For linear elastic, or Hookean solid $\sigma$ is given by: 
$\sigma=2 \mu \varepsilon+\lambda \operatorname{tr}(\varepsilon) I$

and for the linear viscous or Newtonian fluid is given by:

$\sigma=2 \eta \operatorname{dev}(\dot{\varepsilon})-p I$

where $\varepsilon$ is the strain tensor, $\dot{\varepsilon}$ is the strain rate tensor and $\operatorname{dev}($.$) is the deviatoric part of the a$ tensor.

The density and pressure for both liquids and solids are linked with the barotropic relation:

$\frac{\partial \rho}{\partial p}=\frac{\rho}{K}$

where $\mathrm{K}$ is the bulk modulus. The definition of pressure for solids will be given later on. For small variations in pressure about a reference pressure $\mathrm{p}_{0}$, equation (5) can be linearised as:

$\rho \approx \rho_{0}\left[1+\left(p-p_{0}\right) / K\right]$

In order to get to a single set of equations, the stress tensor for the solids is written as a function of velocity and pressure. This is done in two steps: in the first step we create a velocity formulation from the standard displacement formulation and in the second we extract pressure by splitting the stress tensor to its deviatoric and hydrostatic part.

\section{Displacement formulation for solids}

Using the definition of the strain tensor $\varepsilon=1 / 2\left(\nabla D+\nabla D^{T}\right)$ and substituting in equation (3), we obtain the stress tensor is as a function of displacement:

$\sigma=\mu \nabla D+\mu(\nabla D)^{T}+\lambda \operatorname{tr}(\nabla D) I$

The momentum equation can then be written as:

$\frac{\partial \rho\left(\frac{\partial D}{\partial t}\right)}{\partial t}=\nabla \cdot\left[\mu \nabla D+\mu(\nabla D)^{T}+\lambda \operatorname{tr}(\nabla D) I\right]$

This is the standard equation for displacement that is used in stress analysis for solids. In order to derive equation (8), it was assumed that the convection term in equation (1) is negligible for small deformations.

\section{Velocity formulation for solids}

Displacement can be obtained by integrating the velocity using any integration method. In the present paper we use the trapezoidal rule (see Figure 2)

$D=\int_{t_{0}}^{t+\Delta t} U d t=D_{\Sigma}+\frac{\Delta t}{2}\left[U^{n}+U^{o}\right]$

where the superscripts (n) and (o) denote the new and old time step respectively. Substituting (9) into equation (8), we obtain a velocity- based formulation:

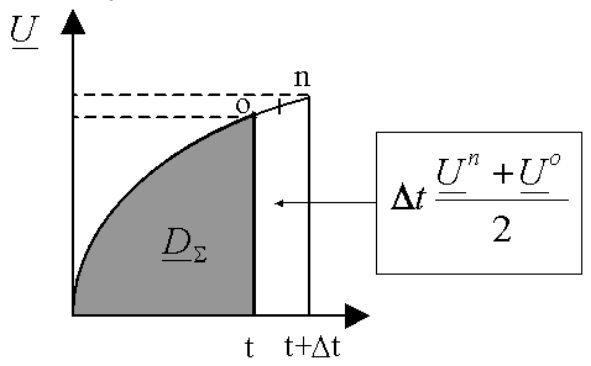

Figure 2: Integration of velocity

$\frac{\partial \rho U}{\partial t}=\frac{\Delta t}{2} \nabla \cdot\left[\mu \nabla U+\mu(\nabla U)^{T}+\lambda \operatorname{tr}(\nabla U) I\right]+\nabla \cdot \Sigma^{+}$

where the tensor $\Sigma^{+}$contains the contribution of the 'old' time instant. This is the end of the first step mentioned earlier. The next step is to extract pressure. 


\section{Velocity-pressure formulation for solids}

The stress tensor $\sigma$ can be split to its deviatioric and hydrostatic part:

$\sigma=\operatorname{dev}(\sigma)-p I$

i.e. pressure is defined as $p=-\frac{1}{3} \operatorname{tr}(\sigma)$. This definition of pressure has been used in the past [8] in order to handle incompressible solids and to avoid the node-locking problem. Substituting into the momentum equation we get:

$\frac{\partial \rho U}{\partial t}=\frac{\Delta t}{2} \nabla \cdot\left[\mu \nabla U+\mu(\nabla U)^{T}+\frac{2}{3} \mu t r(\nabla U) I\right]+\nabla \cdot \operatorname{dev} \Sigma^{+}+\nabla p$

This is the final form of the momentum equation for solids that contains velocity and pressure as unknowns.

\section{DISCRETISATION METHOD}

The system of momentum and continuity equations needs to be solved numerically for the primitive variables of velocity and pressure. The finite volume (FV) method has been widely used in computational fluid dynamics for the solution Navier-Stokes equations but only in recent years in the area of stress analysis. However, since we seek a unified solution method, the finite volume method is used here for solids as well.

The momentum equation in a semidiscretised form can be written as:

$\alpha_{P} U_{P}=H(U)-\nabla p$

where $\mathrm{H}(\mathrm{U})$ contains the contributions of the surrounding nodes as well as all other source terms except pressure. Substituting equation (13) and equation (6) into the continuity equation (2) and setting $\psi=\rho_{0} / K$, a new PDE for pressure can be obtained:

$\frac{\partial \psi p}{\partial t}+\nabla \cdot\left[\left(\rho_{0}-\psi p_{0}\right) \frac{H(U)}{\alpha_{P}}\right]+\nabla \cdot\left[\psi p \frac{H(U)}{\alpha_{P}}\right]-\nabla \cdot\left[\frac{\rho}{\alpha_{P}} \nabla p\right]=0$

\section{Velocity-pressure coupling}

In order to ensure that the velocity field satisfies the continuity equation, the Pressure Implicit with Splitting of Operators (PISO) algorithm was adopted [9]. The PISO algorithm is most suitable for transient problems and can be used for compressible flows in an iterative manner. This solver has been widely used in fluids, but it can now be used for solids as well since we have the same pressure-velocity formulation.

\section{Boundary conditions}

The implementation of the boundary conditions for the fluids is well known. On the other hand care should be taken in the choice of boundary conditions for solids. The solid requires a traction boundary for pressure and velocity that can be obtained by applying force balance at the boundary.

\section{STABILITY ANALYSIS}

Having reformulated the equations for solids to resemble those of fluids and having selected a single discretisation method for both components, the next step is the selection of appropriate time marching and spatial discretisation schemes. In finite elements, one of the most widely used time advancement schemes for structural dynamic problems is the Newmark scheme which can provide very accurate and non-dissipative results if its coefficients are appropriately chosen. In the present paper we select time and spatial discretisation schemes for the reformulated equations for solids that are identical with those widely used for the transient fluids equations. 
The purpose of this section is to assess the stability, accuracy and dissipation characteristics of these schemes when applied to the solids equations.

A number of methods exist to investigate the stability limits of a discretisation scheme. One such method is the Fourier or Von Neuman analysis [10]. This method is used to investigate the stability of the numerical method for the solution of the one-dimensional displacement equations used in the standard stress analysis and the one dimensional velocity equation developed and used in this paper.

\section{Displacement formulation for solids}

The standard stress analysis equation in one-dimension with the assumption of constant density is written as:

$\frac{\partial^{2} D_{x}}{\partial t^{2}}=c^{2} \frac{\partial^{2} D_{x}}{\partial t^{2}}, \quad t \in[0, \infty), x \in[0, L]$

where $\mathrm{c}$ is the wave speed. This is the standard $1 \mathrm{D}$ wave equation. Using the first order Euler implicit difference approximation to approximate the second order time derivative and the second order central approximation for the space derivatives one obtains:

$D_{j}^{n}-2 D_{j}^{n-1}+D_{j}^{n-2}=C_{0}{ }^{2}\left(D_{j-1}^{n}-2 D_{j}^{n}+D_{j+1}^{n}\right)$

where the Courant number is $C_{0}=c \Delta t / \Delta x$. The stencil of this Euler implicit scheme is shown in Figure 3a.

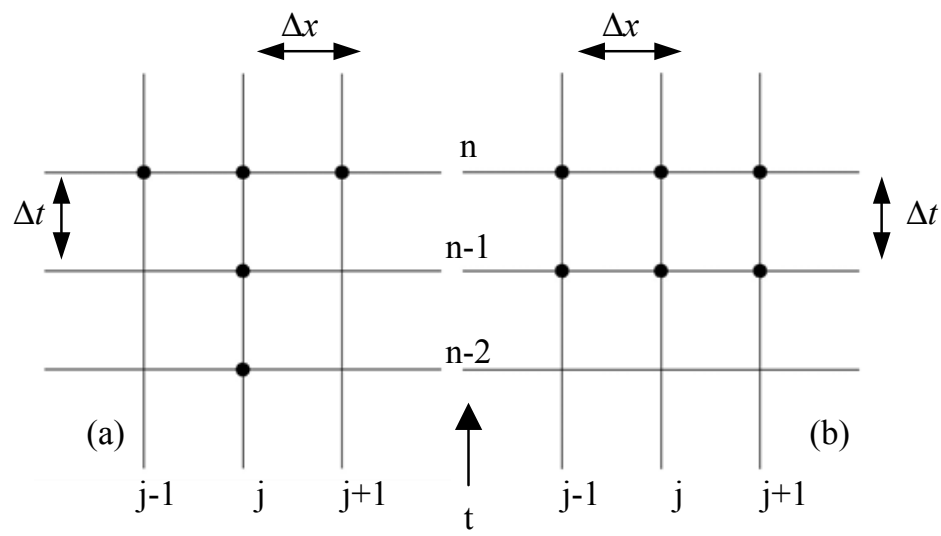

Figure 3: (a) Stencil for the 1D displacement formulation and (b) stencil of the 1D velocity formulation.

It is assumed that the solution of the finite difference scheme can be written as a Fourier series in complex, exponential form for any time level, $n$. After substitution, factorization and cancellation of the common terms the characteristic or dispersion equation is obtained:

$\left(1+4 C_{0}^{2} \sin ^{2} \frac{\alpha}{2}\right) \lambda^{2}-2 \lambda+1=0$

where $\lambda$ is the amplification factor between two consecutive time steps, $\alpha=2 \pi / N$ and $N \in[2, \infty)$. Equation 17 has two solutions, therefore two amplification factors exist that must satisfy the stability condition, although the exact solution has a single value of the amplification. The solution with the positive sign corresponds to the physical solution, whereas the one with the negative sign propagates in the other direction. The solution of Equation 17 is:

$$
G=\frac{1}{1 \pm i 2 C_{0} \sin \frac{\alpha}{2}}
$$

\section{Velocity formulation for solids}

The one-dimensional velocity formulation for solids, with the assumption of constant density, is equivalent to the following system of first order ordinary differential equations: 


$$
\begin{aligned}
& \frac{\partial U_{x}}{\partial t}=c^{2} \frac{\partial^{2} D_{x}}{\partial x^{2}} \\
& \frac{\partial D_{x}}{\partial t}=U_{x}
\end{aligned}
$$

For equation (19), the first order Euler implicit difference approximation is used to approximate the first order time derivative and for the space derivatives the second order central approximation. These choices for the time marching and spatial discretisation schemes are very popular for the equations for fluids. The trapezoidal rule is used for the approximation of the integral in equation (20), as already mentioned. The system of Equations (19) and (20) after substitution of time and space approximations can be written as:

$$
\begin{aligned}
& D_{j}^{n}-D_{j}^{n-1}=\frac{\Delta t}{2}\left(U_{j}^{n}+U_{j}^{n-1}\right) \\
& U_{j}^{n}-U_{j}^{n-1}=\frac{C_{0}^{2}}{\Delta t}\left(D_{j-1}^{n}-2 D_{j}^{n}+D_{j+1}^{n}\right)
\end{aligned}
$$

The stencil of this system of equations is shown in Figure 3b. It is assumed that the solution of the finite difference scheme can be written as a Fourier series in complex, exponential form for any time level, $n$. However, since we have now two equations, the stability is determined by the eigenvalues of the amplification matrix. After substitution, factorization and cancellation of the common terms the characteristic or dispersion equation for the eigenvalues is obtained:

$$
\lambda^{2}-\frac{2\left(1-C_{0}^{2} \sin ^{2} \frac{\alpha}{2}\right)}{1+2 C_{0}^{2} \sin ^{2} \frac{\alpha}{2}} \lambda+\frac{1}{1-2 C_{0}^{2} \sin ^{2} \frac{a}{2}}=0
$$

where $\alpha=2 \pi / N$ and $N \in[2, \propto)$. The solution with the positive sign corresponds to the physical solution, whereas the one with the negative sign propagates in the other direction. The analytical solution of Equation (23) is:

$$
G=\frac{1}{-2-2 C_{0}^{2} \sin ^{2} \frac{\alpha}{2} \pm \sqrt{2 C_{0}^{2} \sin ^{2} \frac{\alpha}{2}\left(2 C_{0}{ }^{2} \sin ^{2} \frac{\alpha}{2}+8\right)}}
$$

The amplitude portraits for different Courant numbers $C_{0}=1 / 4,1 / 2,3 / 4,1,5 / 4$ in comparison with those of the displacement equation are shown in the following Figure 4.
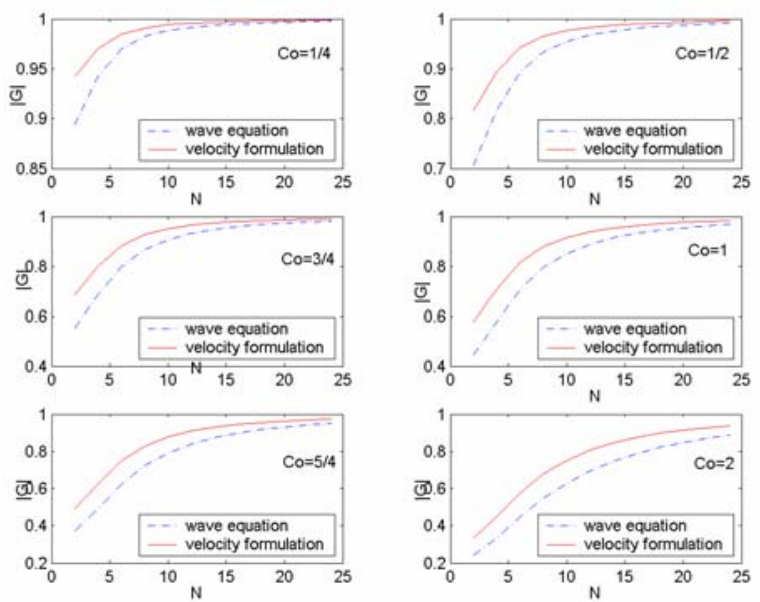

Figure 4: Comparison of amplitude portraits for the 1D velocity and displacement formulations. 
It can be seen from figure 4 that the time and spatial discretisation schemes used for the velocity formulation lead to an unconditionally stable method for all $C_{0}$. The numerical damping (dissipation) is also smaller compared to the one added by the scheme used for the displacement equation.

In the following section the aforementioned schemes are applied for the solution of a two dimensional structural dynamic problem and the results are discussed in the light of the previous 1D linear stability analysis.

\section{VALIDATION OF THE RESULTS}

The mathematical model and the solution method are standard for compressible or incompressible fluid modeling, but as they are new for solids it needs to be tested.

A transient beam-bending case is chosen to test the method. This case was chosen because apart from normal stress it comprises also shear stress. The effect of shear stress is of great importance in wave propagation, thus such a case would be a good validation tool. Figure 5 shows a schematic representation of the case considered.

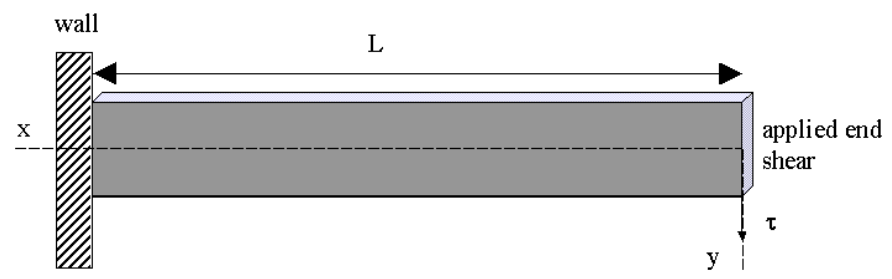

Figure 5: 2D beam bending case.

The cantilevered beam is deflected from the horizontal position by applying at the right end a shear stress $\tau=10 \mathrm{kPa}$ and is then released. The upper and lower surfaces of the beam are free traction boundaries. The beam specifications can be seen in Table 1 .

\begin{tabular}{|ll|}
\hline Property & Value \\
\hline Modulus $E$ & $4 \times 10^{9} \mathrm{~Pa}$ \\
Poisson's ratio $v$ & 0.3 \\
Density $\rho$ & $1450 \mathrm{Kg} / \mathrm{m}^{3}$ \\
Length $L$ & $20 \mathrm{~m}$ \\
Height $h$ & $5 \mathrm{~m}$ \\
Depth $w$ & $1 \mathrm{~m}$ \\
\hline
\end{tabular}

Table 1: Material properties and dimensions of the beam.

A two-dimentional analytical solution for the steady state of the maximum end displacement at $\mathrm{x}=0$ is given by [11]:

$\delta=\frac{4 \tau}{\mathrm{E}} \frac{L^{3}}{h^{2}}\left[1+\frac{3}{4}(1+v)\left(\frac{h}{L}\right)^{2}\right]$

In order to calculate the main frequency of oscillation of the beam, a one-dimensional approximation is used for which an analytical solution is available. Unfortunately a two dimensional solution for the frequency has not been found. Thus, the 1D solution is used only as a rough reference guide to validate the computational results. The fundamental eigenfrequency of the undamped oscillation is:

$\omega^{2}=1.875^{4} \frac{E h^{2}}{12 \rho L^{4}}$ 
For the present case, the end displacement is $\delta=0.340 \mathrm{~m}$, the oscillation frequency is $f=$ $\omega / 2 \pi=3.35 \mathrm{~Hz}$ and the propagation of the stress wave through the beam is $c=\sqrt{E / \rho}=1660 \mathrm{~m} / \mathrm{sec}$.

\section{RESULTS}

The time integration scheme used for equation (12) was first-order Euler implicit. A 40x10 mesh was used with a time step $10^{-4} \mathrm{~s}$ corresponding to $C o=0.33$. Convergence was achieved using two PISO loops and four pressure-velocity loops for each time step. The solution gives a sinusoidal oscillation of the beam about the mean static deflection. The beam oscillates with a frequency of $3.32 \mathrm{~Hz}$ and with maximum and mean deflections of $0.62 \mathrm{~m}$ and $0.31 \mathrm{~m}$, respectively. The calculated values are quite close to the values from the analytical solution, which predicts a maximum deflection of $0.68 \mathrm{~m}$ and an oscillation frequency of $3.35 \mathrm{~Hz}$. A comparison between the standard displacement formulation and the new velocity-pressure formulation is shown in Figure 6. For the standard stress analysis formulation, the finite volume method was used using a second-order central discretisation scheme in space and a first-order time integration scheme. The results obtained using the two formulations agree quite well.

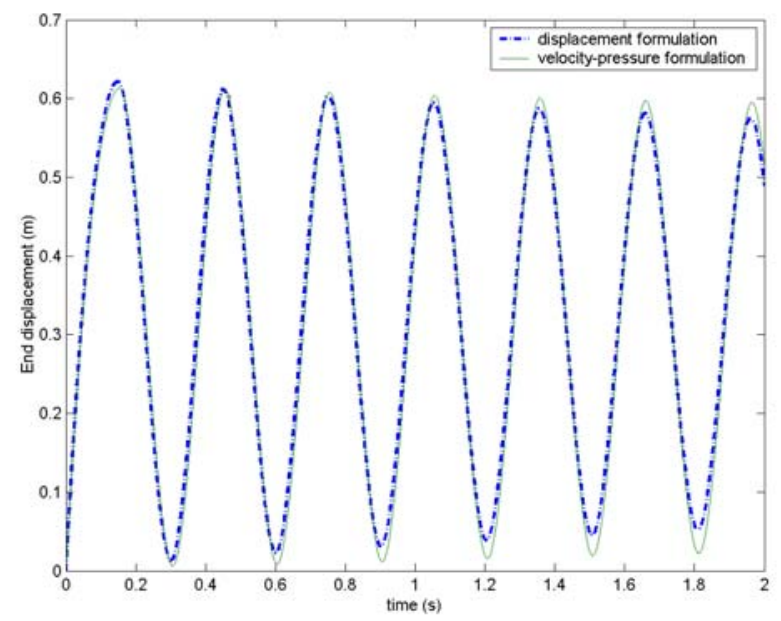

Figure 6: Comparison of end displacement obtained with the standard stress analysis and the velocity-pressure formulation $(\mathrm{Co}=0.33)$.

In order to investigate the numerical dissipation of the two methods, a calculation was performed until time $t=100 \mathrm{~s}$. In a previous study [1], it was found that the standard stress analysis completely dissipates after 56 beam oscillations, whereas the velocity formulation has dissipated $33 \%$ after 332 oscillations. The reason for this behavior can now be explained from the conclusions of linear stability analysis presented earlier on. In Figure 4, it was shown that the velocity formulation is less dissipative compared to the displacement formulation for all Courant numbers. Clearly a more accurate time approximation would reduce the amount of dissipation in the standard formulation. For example, as already mentioned, the Newmark time integration scheme can provide very accurate and non-dissipative results $[8,12]$.

The effect of the discretisation scheme used for the first order time derivative will now be investigated. The discretisation schemes compared are Euler Implicit and Backward Differencing (or three time level method). The latter is a second order accurate scheme and is also frequently used in computational fluid dynamics. In Figure 7 it can be seen that the Backward Differencing scheme is less dissipative that the Euler implicit, as expected. Over a period of 30s (300,000 time steps) the Euler Implicit dissipated about $14.7 \%$ and over $100 \mathrm{~s}(1,000,000$ time steps) about $33.3 \%$. On the other hand the Backward Differencing over a $30 \mathrm{~s}$ period has much smaller dissipation. 


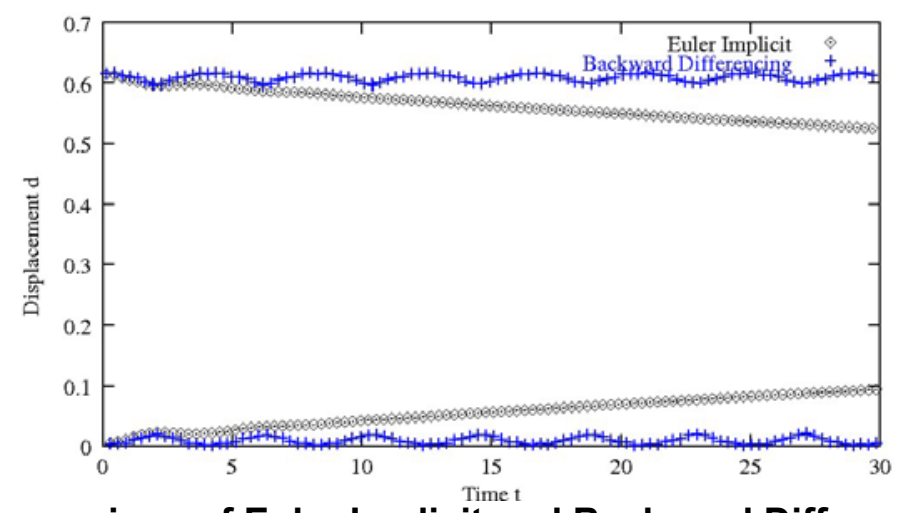

Figure 7: Comparison of Euler Implicit and Backward Differencing scheme (envelope of end displacement).

The accuracy can be improved further with the decrease of the time step size. Figure 8 compares different time step sizes for Euler Implicit discretisation scheme. It can be seen that when the time step is decreased from $10^{-4} \mathrm{~s}$ to $10^{-5} \mathrm{~s}\left(C_{0}=0.033\right)$ the accuracy over a $30 \mathrm{~s}$ period improves about $7.5 \%$. When the time step is decreases from $10^{-5}$ to $10^{-6}\left(C_{0}=0.0033\right)$ there is no significant change, only $0.62 \%$ improvement, but the computational overhead is quite substantial.

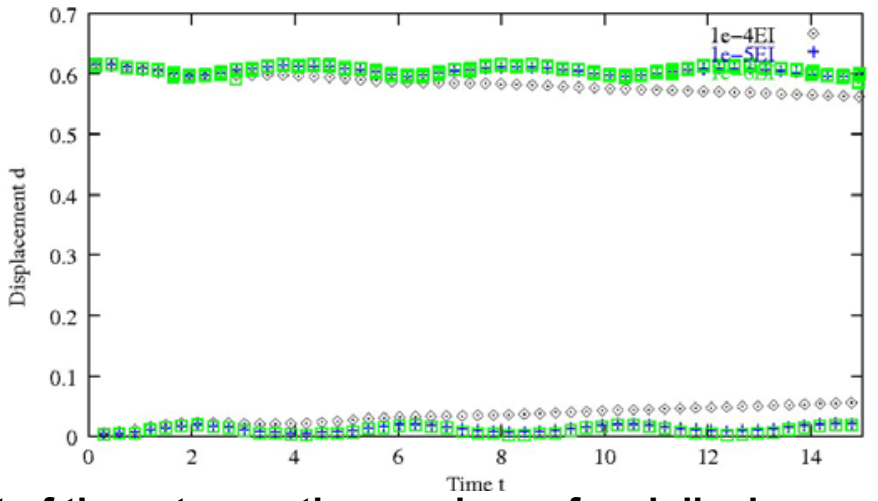

Figure 8: Effect of time step on the envelope of end displacement (Euler Implicit scheme with time steps $10^{-4} \mathrm{~s}, 10^{-5} \mathrm{~s}$ and $10^{-6} \mathrm{~s}$ ).

The results of the first order accurate Euler Implicit scheme with a decreased time step can be compared with those of the second order accurate Backward Differencing scheme.

Figure 9 illustrates that for a period of 30s the results of Backward Differencing with time step $10^{-4} \mathrm{~s}$ and Euler Implicit with time step $10^{-5} \mathrm{~s}$ are almost identical. The Backward differencing scheme with $10^{-4} \mathrm{~s}$ time step is $1.2 \%$ less dissipative than the Euler Implicit with $10^{-5} \mathrm{~s}$ time step.

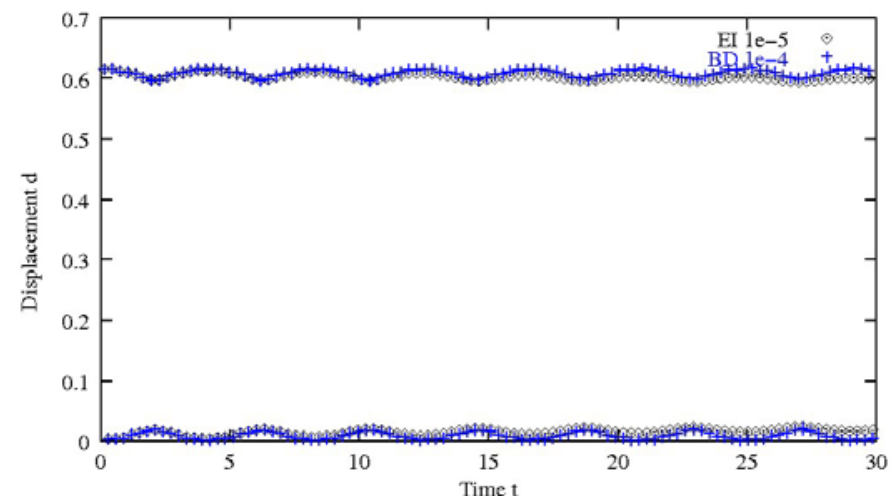

Figure 9: Comparison of envelop of end displacement obtained with Euler Implicit (time step $10^{-5} \mathrm{~s}$ ) and Backward differencing (time step $10^{-4} \mathrm{~s}$ ). 
In figure 9, the envelope of the Backward Differencing scheme exhibits repeatable beats. The beats can be either physical or numerical and related to the discretisation of the computational domain. If these beats are physical they can only represent the first eigenmode of the vibration. In order to investigate this, the two-dimensional beam bending case was run using ANSYS finite element commercial package. The beats appearing in the envelope of the displacement of our discretisation have a frequency of $0.2369 \mathrm{~Hz}$, where the frequency of the first mode found by ANSYS is $0.677 \mathrm{~Hz}$. Thus, it can be concluded that their appearance is of numerical nature. In

Figure 9, the beats are independent of time step size.

The effect of mesh size on the beats was also investigated. The mesh resolution presented up to now has been $40 \times 10$ cells. In the third direction there is always one cell. As this is a twodimensional investigation, displacement and velocity are not computed in the third direction. For the other meshes use the time step size is kept the same $\Delta t=10^{-4} \mathrm{~s}$ resulting in a $C_{0}=0.33$ and the discretisation scheme for the temporal term is Backward differencing. The different mesh resolutions applied were $60 \times 20$ and 200x50 cells in $x$ and $y$ direction respectively. The results are presented in Figure 10.

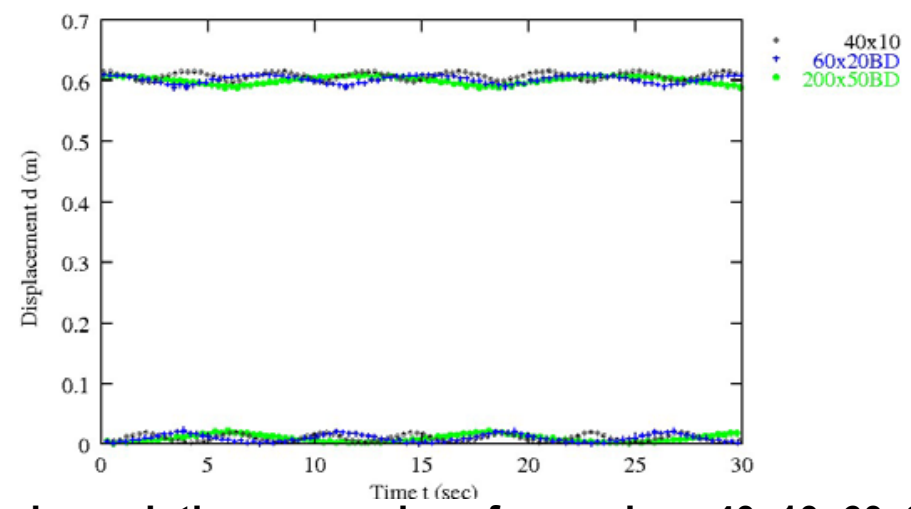

Figure 10: Mesh resolution comparison for meshes: 40x10, 60x20 and 200x50 cells.

Beats appear in the displacement envelope in all cases compared, but their number decreases with the increase of the mesh resolution. The frequency of the beats in the $40 \times 10$ cells mesh is $0.1148 \mathrm{~Hz}$, while for the $60 \times 20$ reduces to $0.1309 \mathrm{~Hz}$ and for $200 \times 50$ drops down to $0.0782 \mathrm{~Hz}$, thus almost disappear (only two beats in 300,000 time steps corresponding to 100 oscillations).

A parametric study was also undertaken with different beam dimensions and end load. The conditions, the predicted and analytic results as well as their difference are shown in the following table 2 .

\begin{tabular}{|c|c|c|c|}
\hline Variable & Analytical & Predicted & $\%$ Difference \\
\hline \multicolumn{4}{|c|}{ Beam size: $10 m \times 5 m ;$ end shear: 1 e6 Pa } \\
\hline Max Dispacement [m] & 0.0995 & 0.0866 & 12.96 \\
\hline Frequency [Hz] & 13.41 & 11.64 & 13.29 \\
\hline \multicolumn{4}{|c|}{ Beam size: $20 m \times 5 m$; end shear: 1 e6 $\mathrm{Pa}$} \\
\hline Max Dispacement [m] & 0.68 & 0.62 & 8.82 \\
\hline Frequency $[\mathrm{Hz}]$ & 3.35 & 3.32 & 0.9 \\
\hline \multicolumn{4}{|c|}{ Beam size: $40 m \times 5 m$; end shear: 1 e6 $\mathrm{Pa}$} \\
\hline Max Dispacement [m] & 5.2 & 4.72 & 9.21 \\
\hline Frequency [Hz] & 0.84 & 0.86 & 2.05 \\
\hline \multicolumn{4}{|c|}{ Beam size: $20 \mathrm{mx} 5 \mathrm{~m}$; end shear: 5 e5 $\mathrm{Pa}$} \\
\hline Max Dispacement [m] & 0.34 & 0.313 & 7.94 \\
\hline Frequency $[\mathrm{Hz}]$ & 3.35 & 3.29 & 1.79 \\
\hline
\end{tabular}

Table 2: Comparison between analytical and computational solutions for beams with different dimensions and loading conditions. 
In all cases the time step used was $\Delta t=10^{-4} \mathrm{~s}$, the temporal discretisation scheme applied was Backward differencing and the mesh resolution was kept equivalent with the beam length i.e. constant $\Delta x$. For example, for the case with $10 \mathrm{~m}$ beam length the mesh used was $20 x 10$ cells. The end displacement is shown in figure 11. The percentage differences between the analytic solution and the predictions for this case are slightly above $10 \%$ as shown in Table 2 . It must be noted however that as the analytical solution is $1 \mathrm{D}$, the shorter the beam is in relation to its height, the less accurate the solution would be.

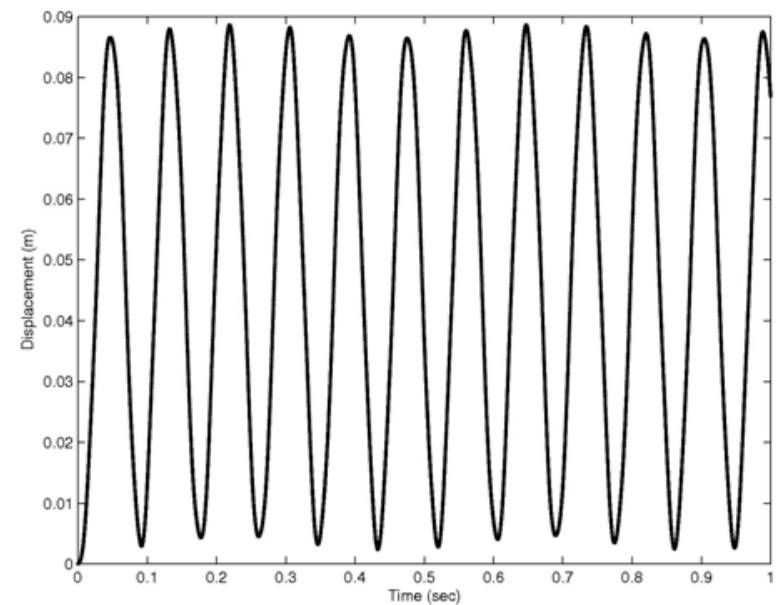

Figure 11: End displacement for a beam with size $10 \mathrm{~m} \times 5 \mathrm{~m}$.

For the case with double the beam length $(40 \mathrm{mx} 5 \mathrm{~m})$ the mesh used was $80 \mathrm{x} 10$ cells (Figure 12). The end displacement was $4.72 \mathrm{~m}$ and the frequency was $0.86 \mathrm{~Hz}$. The percentage difference between the analytical and the numerical solution is $2.05 \%$ and for the displacement $9.21 \%$ (Table 2).

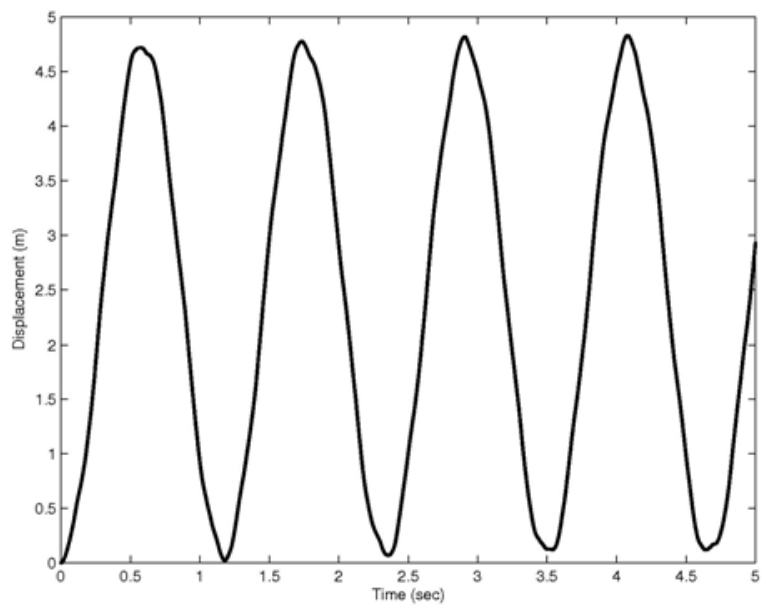

Figure 12: Beam with size $40 \mathrm{~m} \times 5 \mathrm{~m}$.

In the case where the applied end shear was halved $\tau=5 \mathrm{e} 5 \mathrm{~Pa}$ the mesh resolution was $40 \times 10$ cells, the same as the one in the base case. Figure 13 presents the results. The maximum end displacement is $0.313 \mathrm{~m}$ and the frequency of the oscillation of the beam is $3.29 \mathrm{~Hz}$ i.e. the frequency does not depend on the applied load. The percentage difference for the frequency between the analytical solution and the numerical solution is $1.79 \%$ and for the maximum displacement is $7.94 \%$ (Table 2). 


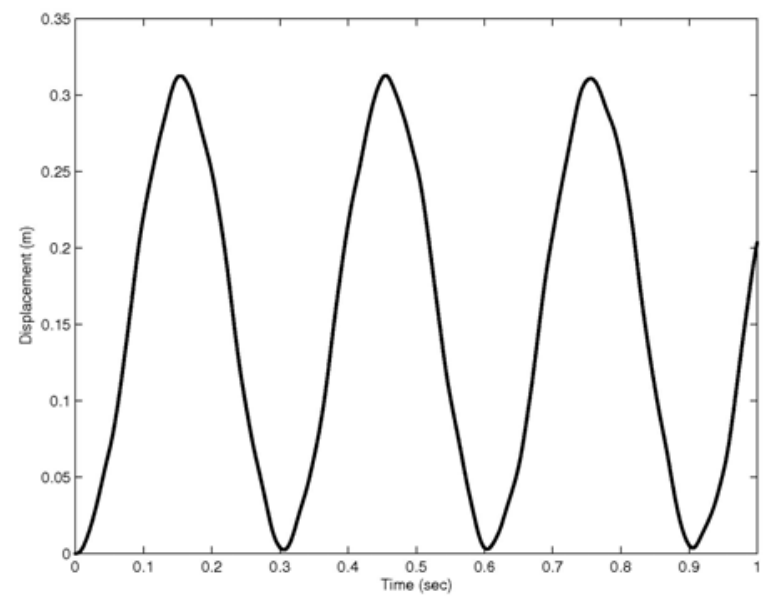

Figure 13: Beam with size $20 \mathrm{~m} \times 5 \mathrm{~m}$ and with applied end shear $\tau=5 \mathrm{e} 5 \mathrm{~Pa}$.

\section{IMPLEMENTATION IN FSI}

The method can be directly used in a fluid-structure interaction problem to create a monolithic algorithm where a single mesh and a unified method is used for both fluids and solids. The grid cells will hold an extra property defining the phase of the medium, i.e. whether it is a fluid or a solid. In that way according to the phase the appropriate material properties associated with the state of the continuum will be used.

\section{CONCLUSIONS}

The single solution method $[1,7]$ suitable for fluid-structure interaction problems is general, with no approximations made to the basic continuum model for Hookean solids and Newtonian fluids.

The principal idea is that the fluid and structural analysis is performed by a single set of equations in a single solution domain, in a fully implicit and coupled manner. The single set of equations has as primitive variables velocity and pressure. The pressure-velocity coupling is handled using the PISO algorithm. Note also that since pressure is used as an independent variable, the study of structural deformations of fully incompressible solids is straightforward while well-known problems such as node locking [13] are avoided.

The unified solution method for FSI was validated in this paper against a transient beam bending case. The discretisation method was stable and less dissipative than the standard stress analysis descretisation method used. This was in agreement with the one-dimensional linear stability analysis.

The comparison of the Backward differencing with Euler implicit for the discretisation of the temporal term showed that the former introduces smaller amounts of dissipation and gives more accurate results. The decrease of the time step size by a factor of 10 for the Euler implicit improves the accuracy and brings the results closer to these of Backward differencing.

The results showed that the unified solution method is capable of solving standard solid mechanics problems with sufficient accuracy. The next step of this work is the application of this method to full FSI problems. 


\section{ACKNOWLEDGMENTS}

The authors would like to thank EPSRC (research grant GR/N65769) for funding this project and Nabla Ltd.

\section{REFERENCES}

[1] Giannopapa, C. G. and Papadakis, G., 2004, "A new formulation for solids suitable for a unified solution method for fluid-structure interaction problems," ASME PVP 2004, San Diego California, July , PVP-Vol. 491-1, pp. 111-117.

[2] Wiggert, D. C., and Tijsseling, A. S., 2001, "Fluid transients and fluid-structure interaction in flexible liquid-filled piping," ASME Applied Mechanics Reviews, 54, pp. 455-481.

[3] Korterweg, D. J., 1878, "Uber die Fortplanzungs-geschwindigkeit des Schalles in elastischen rohen", Annalen der Physik und Chemie, 5, pp 525-542.

[4] Felippa, C. A., Park, K. C. and Farhat, F., 2001, "Partitioned analysis of coupled mechanical systems. Computer methods in applied mechanics and engineering," 190, pp. 3247-3270.

[5] Hübner, B., Walhorn, E., and Dinkler, D., 2004, "A monolithic approach to fluid-structure interaction using space-time finite elements," Computer methods in applied mechanics and engineering, 193, pp. 2087-2104.

[6] van Brummelen, E. H. and Koren, B., 2003, "A pressure-invariant conservation godonov-type method for barotropic two-flud flows," Journal of computational physics, 185, pp. 289-308.

[7] Giannopapa, C. G., 2004, Fluid-Structure interaction in flexible vessels, PhD thesis, University of London.

[8] Bathe, K. J., 1997, Finite element procedures. Prentice Hall, Englewood Cliffs, New Jersey.

[9] Issa, R. I., 1986, "Solution of the implicit discretised fluid flow equation by operatorsplitting", Journal of Computational Physics, 62, pp 40-65.

[10] Mattheij, R. M. M., Rienstra, S. W., and tenTije Boonkkamp, J. H. M., 2005, Partial differential equations: modeling, analysis and computing, SIAM, Philadelphia (in Press).

[11] Geradin, M., and Rixen, D., 1997, Mechanical vibrations, Wiley, Chichester, UK.

[12] Sloan, A. K., Bailey, C., and Cross, M., "Dynamic solid mechanics using the finite volume methods," Applied mathematical modeling, 27, pp. 69-87.

[13] Bath, M., and Kamm, R. D., 1999, "A fluid structure interaction finite element analysis of pulsatile blood flow in arterial structures", Journal of Biomechanical Engineering-Transactions of AMSE, 121, pp.361-369. 